\title{
On Finite Time Singularity and Global Regularity of an Axisymmetric Model for the 3D Euler Equations
}

\author{
Thomas Y. Hou* $\quad$ Zhen Lei $^{\dagger} \quad$ Shu Wang ${ }^{\ddagger} \quad$ Chen Zou $^{\S}$
}

November 25, 2013

\begin{abstract}
We investigate the large time behavior of an axisymmetric model for the 3D Euler equations. In [22], Hou and Lei proposed a 3D model for the axisymmetric incompressible Euler and Navier-Stokes equations with swirl. This model shares many properties of the 3D incompressible Euler and Navier-Stokes equations. The main difference between the 3D model of Hou and Lei and the reformulated 3D Euler and Navier-Stokes equations is that the convection term is neglected in the 3D model. In [24, the authors proved that the 3D inviscid model can develop a finite time singularity starting from smooth initial data on a rectangular domain. A global well-posedness result was also proved for a class of smooth initial data under some smallness condition. The analysis in [24] does not apply to the case when the domain is axisymmetric and unbounded in the radial direction. In this paper, we prove that the 3D inviscid model with an appropriate NeumannRobin boundary condition will develop a finite time singularity starting from smooth initial data in an axisymmetric domain. Moreover, we prove that the 3D inviscid model has globally smooth solutions for a class of large smooth initial data with some appropriate boundary condition.
\end{abstract}

\section{Introduction}

Whether the 3D incompressible Navier-Stokes equations can develop a finite time singularity from smooth initial data with finite energy is one of the most challenging questions in nonlinear partial differential equations [14. There have been many previous studies devoted to this challenging question, see e.g. [7, 5, 9, 11, 12, 6] and two

\footnotetext{
${ }^{*}$ Computing \& Mathematical Sciences, California Institute of Technology, Pasadena, CA 91125, USA

${ }^{\dagger}$ School of Mathematical Sciences; LMNS and Shanghai Key Laboratory for Contemporary Applied Mathematics, Fudan University, Shanghai 200433, P. R. China.

${ }_{\ddagger}^{\ddagger}$ College of Applied Sciences, Beijing University of Technology, Beijing 100124, China.

${ }^{\S}$ Department of Mechanics and Aerospace Engineering, COE, Peking University, Beijing 100871, China
} 
recent review articles [1, 8]. There have been a number of attempts to investigate possible finite time singularity formation of the 3D Euler equations numerically, see e.g. [16, 27, 3]. So far, the numerical evidence for a finite time blow-up is not yet conclusive [19, 18, 17.

Global regularity results for the 3D Navier-Stokes equations have been obtained using energy estimates under some smallness assumption on the initial data [26, 29, 25]. It is well known that the convection term does not play an essential role in energy estimates due to the incompressibility of the velocity field. Thus, it is not clear how convection may contribute to global well-posedness of the 3D incompressible NavierStokes equations.

In [21, Hou and Li investigated the stabilizing effect of convection via an exact $1 \mathrm{D}$ model. They found that the convection term plays an essential role in canceling the destablizing vortex stretching terms in this $1 \mathrm{D}$ model. This observation enabled them to obtain a pointwise estimate via a Liapunov function which controls the dynamic growth of the derivative of vorticity. Motivated by the work of [21], Hou and Lei further investigated the role of convection by constructing the following 3D model of the axisymmetric Navier-Stokes equations with swirl [22]:

$$
\left\{\begin{array}{l}
\partial_{t} u=\nu\left(\partial_{r}^{2}+\frac{3}{r} \partial_{r}+\partial_{z}^{2}\right) u+2 u \partial_{z} \psi \\
\partial_{t} \omega=\nu\left(\partial_{r}^{2}+\frac{3}{r} \partial_{r}+\partial_{z}^{2}\right) \omega+\partial_{z}\left(u^{2}\right) \\
-\left(\partial_{r}^{2}+\frac{3}{r} \partial_{r}+\partial_{z}^{2}\right) \psi=\omega .
\end{array}\right.
$$

When $\nu=0$, we refer to the above model as the 3D inviscid model. This model derived by using a reformulated Navier-Stokes equations in terms of a set of new variables $(u, \omega, \psi)=\left(u^{\theta}, \omega^{\theta}, \psi^{\theta}\right) / r$, where $r=\sqrt{x_{1}^{2}+x_{2}^{2}}, u^{\theta}$ is the angular velocity, $\omega^{\theta}$ the angular vorticity, and $\psi^{\theta}$ the angular stream function, see [21, 22]. The only difference between this 3D model and the reformulated Navier-Stokes equations is that we neglect the convection term in the model. This new 3D model shares several well known properties of the full 3D Euler or Navier-Stokes equations [22]. These include an energy identity for smooth solutions, an artificial incompressible constraint, a nonblowup criterion of Beale-Kato-Majda type [2], a non-blowup criterion of Prodi-Serrin type [30, 31], and a partial regularity result for the model [23], which is an analogue of the Caffarelli-Kohn-Nirenberg theory [4, 28] for the full Navier-Stokes equations.

Despite the striking similarity at the theoretical level, this 3D model seems to have a very different behavior from that of the Euler or Navier-Stokes equations. Numerical study in [22] seems to suggest that the model develop a potential finite time singularity from smooth initial data with finite energy. However, the mechanism that leads to the singular behavior of the 3D model seems to be destroyed when the convection term is added back to the model. In a recent paper [24], Hou, Shi and Wang proved rigorously that the inviscid model can indeed develop a finite time singularity for a class of smooth initial data with some Neumann-Robin boundary condition. Moreover, they proved a global well-posedness result for a class of small smooth initial data. The analysis in [24] 
was carried out for a rectangular domain, which does not apply to the axisymmetric domain considered in this paper.

In this paper, we prove that the 3D inviscid model with some appropriate boundary conditions of Neumann-Robin type can develop a finite time singularity starting from smooth initial data on a bounded or an unbounded exterior axisymmetric domain. Moreover, we obtain a global well-posedness result for a class of large smooth initial data under some Dirichlet boundary condition.

Our analysis in this paper is similar in spirit to that of [24]. However, there are several new ingredients in this work. The first one is that our blowup result applies to a bounded or unbounded exterior domain in the axisymmetric geometry. The local well-posedness of the 3D model with a boundary condition of the Neumann-Robin type is more complicated than the case considered in [24] and involves the use of the modified Bessel function. The second ingredient is to construct a special positive test function that satisfies several requirements. In the case of a bounded domain, we use the first eigenfunction of the Laplacian operator with homogeneous Dirichlet boundary condition. In the case of an unbounded exterior domain, a positive and decaying eigenfunction of the Laplacian operator does not exist. This requires us to relax the constraints on the test function. The third ingredient is an improved estimate which enables us to establish the global regularity of the 3D inviscid model for a class of initial boundary value problem with large initial data.

The paper is organized as follows: In Section 2 we will state our main results and present our main ideas of their proofs. Section 3 is devoted to proving the finite time singularity of the 3D inviscid model. In Section 4 we present our global well-posedness result. Finally, we prove the local well-posedness of the initial boundary value problem in Section 5.

\section{Main Results}

In this section we set up our problem and state our main results. First of all, let us recall that the $3 \mathrm{D}$ model (1.1) is formulated in terms of a set of new variables $u=\frac{u^{\theta}}{r}, \omega=\frac{\omega^{\theta}}{r}, \psi=\frac{\psi^{\theta}}{r}$. Due to this change of variables, the original three-dimensional Laplacian operator for $\psi^{\theta}$ has been changed into a five-dimensional Laplacian operator for $\psi$ in the axisymmetric cylindrical coordinate in (1.1). Thus we can reformulate our 3D inviscid model in the three-dimensional axisymmetric cylindrical domain

$$
D\left(\gamma_{1}, \gamma_{2}\right)=\left\{\left(x_{1}, x_{2}, z\right) \in \mathbb{R}^{3}: \gamma_{1} \leq r=\sqrt{x_{1}^{2}+x_{2}^{2}}<\gamma_{2} ; 0 \leq z \leq 1\right\}
$$

as a five-dimensional problem in the axisymmetric cylindrical domain

$$
\Omega\left(\gamma_{1}, \gamma_{2}\right)=\left\{\left(x_{1}, \cdots, x_{4}, z\right) \in \mathbb{R}^{5}: \gamma_{1} \leq r=\left(\sum_{j=1}^{4} x_{j}^{2}\right)^{\frac{1}{2}}<\gamma_{2} ; 0 \leq z \leq 1\right\}
$$


It is much more convenient to perform the well-posedness and the finite time blow-up analysis for our 3D model in the five-dimensional setting. In the remaining part of the paper, we will carry out our analysis in this axisymmetric five-dimensional domain.

Denote

$$
S_{\text {exterior }} \equiv\left\{\beta>0 \quad \mid \quad \beta \neq \frac{k \int_{0}^{\infty} e^{-k \cosh (\theta)} \cosh ^{2}(\theta) d \theta}{\int_{0}^{\infty} e^{-k \cosh (\theta)} \cosh (\theta) d \theta}, \forall k \in \mathbb{Z}^{+}\right\} .
$$

Now we are ready to state the first result of this paper which is concerned with the local well-posedness of classical solutions to the 3D inviscid model with a Neumann-Robin type boundary condition on the exterior domain $\Omega(1, \infty)$.

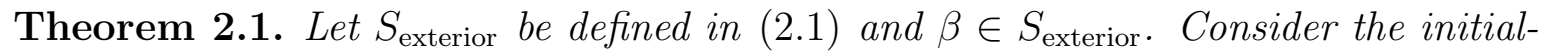
boundary value problem of the $3 D$ model (1.1) with the initial data

$$
\begin{gathered}
u_{0} \in H^{3}(\Omega(1, \infty)), \quad \psi_{0} \in H^{4}(\Omega(1, \infty)), \\
u_{0}^{2}>0 \text { for } z \neq 0 \text { and } z \neq 1,\left.\quad u_{0}\right|_{z=0}=\left.u_{0}\right|_{z=1}=0
\end{gathered}
$$

and the Neumann-Robin type boundary condition

$$
\left.\left(\psi_{r}+\beta \psi\right)\right|_{r=1}=0,\left.\quad \psi_{z}\right|_{z=0}=\left.\psi_{z}\right|_{z=1}=0 .
$$

Then there exists a unique smooth solution $(u, \psi)$ to the $3 D$ inviscid model with the initial data (2.2)-(2.3) and the boundary condition (2.4) on $[0, T)$ for some $T>0$. Moreover, we have

$$
u \in C\left([0, T), H^{3}(\Omega(1, \infty))\right), \quad \psi \in C\left([0, T), H^{4}(\Omega(1, \infty))\right) .
$$

The proof of Theorem 2.1 relies on an important property of the elliptic operator with the mixed Neumann-Robin type boundary condition. Note that the boundary condition in (2.4) is not the third type. To recover the standard elliptic regularity estimate, we need to study the spectral property of the differential operator which

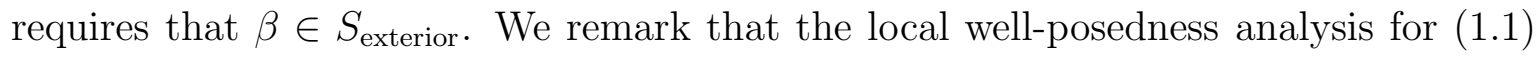
with a mixed Dirichlet-Robin boundary condition has been established in [24] where the non-standard boundary condition is imposed on the axial direction. Here our non-standard boundary condition is imposed on the radial direction. The local wellposedness in such case involves the use of modified Bessel function and is more involved. The proof of Theorem 2.1 will be deferred to Section 5 .

Next, we state the finite time blowup result of the exterior problem (1.1), (2.2), (2.3) and (2.4).

Theorem 2.2. Suppose that all the assumptions in Theorem 2.1 are satisfied. Let $\beta \geq 2+2 \sqrt{1+\frac{\pi^{2}}{4}}, \alpha=\frac{\beta}{2}$ and

$$
\phi(r, z)=\theta(r) \sin (\pi z), \theta(r)=e^{-\alpha r^{2}}, \Phi(r, z)=\phi_{r r}+\frac{3}{r} \phi_{r}+\phi_{z z} .
$$


Then the solution of the $3 D$ inviscid model will develop a finite time singularity in the $H^{3}$ norm provided that

$$
\int_{0}^{1} \int_{1}^{\infty} \Phi \log \left(u_{0}^{2}\right) r^{3} d r d z>0, \quad \int_{0}^{1} \int_{1}^{\infty} \Phi \psi_{0 z} r^{3} d r d z>0
$$

and

$$
\left.\int_{0}^{1} \int_{1}^{\infty} \partial_{z} \psi_{0} \Phi r^{3} d r d z\right)^{2} \geq \frac{16}{c_{0}}\left(\int_{0}^{1} \int_{1}^{\infty}\left(\log u_{0}^{2}\right) \Phi r^{3} d r d z\right)^{3}
$$

where $c_{0}$ is a positive constant defined in (3.14).

The proof of Theorem 2.2 is in spirit similar to that of Theorem 1.1 in [24]. The main ingredient here is to construct a special positive test function which meets our requirements on the unbounded domain case. In [24, the authors can take the product of sine functions in the $x-y$ domain as a testing function since the domain is a rectangular domain. In the case of a unbounded exterior domain in the axisymmetric geometry, a positive and decaying eigenfunction does not seem to exist. This requires us to relax the constraints on the test function. We will present the details of the proof of Theorem 2.2 in Section 3.

Remark 2.3. We remark that similar results in Theorem 2.1 and 2.2 are also true in the bounded domain case $\Omega(0,1)$. We will not present this result here. A more interesting result would be to obtain a similar result for the case of $\Omega(0, \infty)$ with an initial boundary condition whose energy is conserved in time. This would require a different technique. We will report it in a forthcoming paper.

In Theorem 1.1 of [24], the authors proved the finite time singularity of the 3D inviscid model in a rectangular domain. Their Robin boundary condition is imposed on the axial boundary $z=1$. Our next theorem extends their result to the case of a bounded axisymmetric domain $\Omega(0,1)$.

Let $\left(\lambda_{k}, \theta_{k}(r)\right)$ be the eigenvalue-eigenfunction pair of the following Dirichlet eigenvalue problem:

$$
\left\{\begin{array}{l}
-\left(\partial_{r}^{2}+\frac{3}{r} \partial_{r}\right) \theta_{k}=\lambda_{k} \theta_{k}, \quad r \in[0,1) \\
\left.\theta_{k}\right|_{r=1}=0
\end{array}\right.
$$

Define

$$
S_{\text {interior }} \equiv\left\{\beta>0 \mid \beta \neq \lambda_{k}, \beta \neq \frac{\sqrt{\lambda_{k}}\left(e^{\sqrt{\lambda_{k}}}+e^{-\sqrt{\lambda_{k}}}\right)}{e^{\sqrt{\lambda_{k}}}-e^{-\sqrt{\lambda_{k}}}} \text { for all } k=1,2, \cdots\right\},
$$

and

$$
\phi(r, z)=\frac{e^{-\alpha(z-1)}+e^{\alpha(z-1)}}{2} \theta_{1}(r)
$$


Moreover, we assume that $\alpha, \beta$ satisfy

$$
0<\alpha<\sqrt{\lambda_{1}}, \quad \beta=\frac{\lambda_{1}}{\alpha} \frac{e^{\alpha}-e^{-\alpha}}{e^{\alpha}+e^{-\alpha}}>\sqrt{\lambda_{1}}\left(\frac{e^{\sqrt{\lambda_{1}}}+e^{-\sqrt{\lambda_{1}}}}{e^{\sqrt{\lambda_{1}}}-e^{-\sqrt{\lambda_{1}}}}\right) .
$$

We can prove the following local-well-posedness and finite time blow-up results.

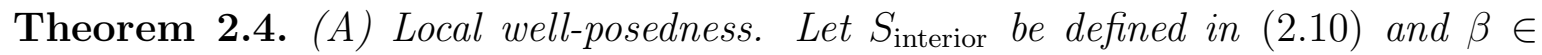
$S_{\text {interior. }}$ Consider the initial-boundary value problem of the $3 D$ model (1.1) with the initial data

$$
u_{0} \in H^{3}(\Omega(0,1)), \quad \psi_{0} \in H^{4}(\Omega(0,1)),\left.\quad u_{0}\right|_{z=0}=\left.u_{0}\right|_{z=1}=0
$$

and the Dirichlet-Robin type boundary condition

$$
\left.\psi\right|_{r=1}=\left.\psi\right|_{z=1}=0, \quad \psi_{z}+\left.\beta \psi\right|_{z=0}=0 .
$$

Then there exists a unique smooth solution $(u, \psi)$ to the $3 D$ inviscid model with the initial data (2.13) and the boundary condition (2.14) on $[0, T)$ for some $T>0$. Moreover, we have

$$
u \in C\left([0, T), H^{3}(\Omega(0,1))\right), \quad \psi \in C\left([0, T), H^{4}(\Omega(0,1))\right) .
$$

(B) Finite time blow-up. Suppose (2.12) is satisfied and $u_{0}^{2}>0$ for $0<z<1$. If $u_{0}$ and $\psi_{0}$ satisfy

$$
\int_{0}^{1} \int_{0}^{1}\left(\log u_{0}^{2}\right) \phi r^{3} \mathrm{~d} r \mathrm{~d} z>0, \quad \int_{0}^{1} \int_{0}^{1} \psi_{0 z} \phi r^{3} \mathrm{~d} r \mathrm{~d} z>0
$$

and

$$
\left(\int_{0}^{1} \int_{0}^{1} \partial_{z} \psi_{0} \phi r^{3} d r d z\right)^{2} \geq \frac{1}{c_{1}}\left(\int_{0}^{1} \int_{0}^{1}\left(\log u_{0}^{2}\right) \phi r^{3} d r d z\right)^{3},
$$

for some absolute constant $c_{1}>0$, then the solution of the $3 D$ inviscid model will develop a finite time singularity in the $H^{3}$ norm.

The local well-posedness of the initial-boundary value problem for $3 \mathrm{D}$ model in Theorem 2.4 can be carried out using almost the same argument as that in [24] by replacing the first eigenfunction $\sin \pi x_{1} \sin \pi x_{2} \sin \pi x_{3} \sin \pi x_{4}$ of the rectangular domain by the first eigenfunction $\phi_{1}(x)$ of the unit ball in $\mathbb{R}^{4}$. The finite time blow-up result can be proved in exactly the same way as in [24]. Since the analysis is essentially the same as that in [24], we will omit the proof of Theorem 2.4 in this paper.

The last theorem extends the global existence result in Theorem 6.1 in [24] to the axisymmetric domain $\Omega(0,1)$. The most interesting aspect of this result is that the initial condition can be made as large as we wish in the Sobolev space. This is achieved by effectively imposing a sufficiently large negative boundary condition for $\left.\partial_{z} \psi\right|_{\partial \Omega(0,1)}=-M$ with $M$ being a large positive constant. 
Theorem 2.5. Let $M>0$ an arbitrarily large constant and $s \geq 3$. Assume that $u_{0} \in H^{s}(\Omega(0,1)), \psi_{0} \in H^{s+1}(\Omega(0,1))$ with $u_{0}(r, 0)=u_{0}(r, 1)=0$ and $\left.\psi_{0}\right|_{r=1}=-M z$, $\left.\partial_{z} \psi_{0}\right|_{z=0}=\left.\partial_{z} \psi_{0}\right|_{z=1}=-M$. Then the $3 D$ inviscid model with the initial and boundary data

$$
u(0, \cdot)=u_{0}(\cdot), \quad \psi(0, \cdot)=\psi_{0}(\cdot)
$$

and

$$
\psi(t, 1, z)=-M z, \partial_{z} \psi(t, r, 0)=\partial_{z} \psi(t, r, 1)=-M
$$

admits a unique global smooth solution $(u, \psi)$ with $u \in C\left([0, \infty) ; H^{s}(\Omega(0,1))\right), \psi \in$ $C\left([0, \infty) ; H^{s+1}(\Omega(0,1))\right)$ provided that

$$
\left\|\nabla \partial_{z} \psi_{0}\right\|_{H^{s-1}} \leq M /\left(8 C_{s}^{2}\right), \quad\left\|u_{0}^{2}\right\|_{H^{s}} \leq M^{2} /\left(4 C_{s}^{3}\right)
$$

where $C_{s}$ is an absolute positive constant depending on $s$ and $\Omega$ only. Moreover, we have

$$
\left\|u(t)^{2}\right\|_{H^{s}(\Omega(0,1))} \leq C_{s}\left\|u_{0}^{2}\right\|_{H^{s}(\Omega(0,1))} e^{-2 M t},
$$

and

$$
\left\|\nabla \partial_{z} \psi\right\|_{H^{s-1}(\Omega(0,1))} \leq\left\|\nabla \partial_{z} \psi_{0}\right\|_{H^{s-1}(\Omega(0,1))}+\frac{C_{s}}{2 M}\left\|u_{0}^{2}\right\|_{H^{s}(\Omega(0,1))}
$$

We will present the proof of Theorem 2.5 in Section 4.

\section{$3 \quad$ Finite Time Singularity in the Exterior Domain}

In this section, we present the proof of Theorem 2.2, which shows that the 3D inviscid model develops a finite time singularity in the exterior domain.

Proof of Theorem 2.2. We will prove Theorem 2.2 by contradiction, as in [24]. By the local well-posedness result, we know that the initial boundary value problem of the $3 \mathrm{D}$ inviscid model has a unique solution $u \in H^{3}(\Omega(1, \infty))$ and $\psi \in H^{4}(\Omega(1, \infty))$ for $0 \leq t \leq T$ for some $T>0$. Let $T_{b}$ be the largest time for which our 3D inviscid model has a unique smooth solution $u \in H^{3}(\Omega)$ and $\psi \in H^{4}(\Omega)$ for $0 \leq t<T_{b}$. We will prove that $T_{b}<\infty$. Suppose that $T_{b}=\infty$. Then we have

$$
u \in C\left([0, \infty), H^{3}(\Omega(1, \infty))\right), \quad \psi \in C\left([0, \infty), H^{4}(\Omega(1, \infty))\right) .
$$

We will prove that (3.1) would lead to a contradiction.

Define $\phi$ and $\Phi$ as in Theorem 2.2. By a straightforward calculation, we have

$$
\Phi(r, z)=\left[4 \alpha^{2} r^{2}-\left(8 \alpha+\pi^{2}\right)\right] \phi(r, z) .
$$

For $\alpha \geq 1+\sqrt{1+\frac{\pi^{2}}{4}}$, it is easy to get that

$$
4 \alpha^{2} r^{2}-\left(8 \alpha+\pi^{2}\right) \geq 4 \alpha^{2}-\left(8 \alpha+\pi^{2}\right) \geq 0 \quad \text { for } r \geq 1
$$


Consequently, we have

$$
\Phi(r, z) \geq 0 \quad \text { for } 1 \leq r, 0 \leq z \leq 1 .
$$

On the other hand, due to the boundary condition on the initial data $u_{0}$ in (2.3), one can use the first equation in (1.1) with $\nu=0$ to solve $u$ :

$$
u^{2}(t, r, z)=u_{0}^{2}(r, z) \exp \left\{4 \int_{0}^{t} \partial_{z} \psi(s, r, z) d s\right\} \quad \text { for } z \neq 0,1 .
$$

Hence, by continuity, as long as the solution is smooth, one also has

$$
u(t, r, 0)=u(t, r, 1)=0 .
$$

Multiplying the second equation in (1.1) with $\nu=0$ by $\phi_{z}$ and integrating over $\Omega(1, \infty)$, we have

$$
-\int_{0}^{1} \int_{1}^{\infty}\left(\partial_{r}^{2}+\frac{3}{r} \partial_{r}+\partial_{z}^{2}\right) \psi_{t} \phi_{z} r^{3} d r d z=\int_{0}^{1} \int_{0}^{\infty} \partial_{z} u^{2} \phi_{z} r^{3} d r d z .
$$

On one hand, using (3.4) and performing integration by parts, we get

$$
\int_{0}^{1} \int_{1}^{\infty} \partial_{z} u^{2} \phi_{z} r^{3} d r d z=-\int_{0}^{1} \int_{1}^{\infty} u^{2} \partial_{z}^{2} \phi r^{3} d r d z=\pi^{2} \int_{0}^{1} \int_{1}^{\infty} u^{2} \phi r^{3} d r d z .
$$

On the other hand, using the boundary condition (2.4) and performing integration by parts, we obtain by noting that $\theta_{r}(1)+\beta \theta(1)=0$ that

$$
\begin{aligned}
- & \int_{0}^{1} \int_{1}^{\infty}\left(\partial_{r}^{2}+\frac{3}{r} \partial_{r}+\partial_{z}^{2}\right) \psi_{t} \phi_{z} r^{3} d r d z \\
= & \left.\frac{d}{d t} \int_{0}^{1}\left(\psi_{r} \phi_{z}-\psi \partial_{r z}^{2} \phi\right)\right|_{r=1} d z-\frac{d}{d t} \int_{0}^{1} \int_{1}^{\infty} \psi\left(\partial_{r}^{2}+\frac{3}{r} \partial_{r}\right) \phi_{z} r^{3} d r d z \\
& +\frac{d}{d t} \int_{0}^{1} \int_{1}^{\infty} \psi_{z} \partial_{z}^{2} \phi r^{3} d r d z \\
= & \left.\pi \theta(1) \frac{d}{d t} \int_{0}^{1}(\cos \pi z)\left(\psi_{r}+\beta \psi\right)\right|_{r=1} d z+\frac{d}{d t} \int_{0}^{1} \int_{1}^{\infty} \psi_{z} \Phi r^{3} d r d z \\
= & \frac{d}{d t} \int_{0}^{1} \int_{1}^{\infty} \psi_{z} \Phi r^{3} d r d z .
\end{aligned}
$$

Combining (3.7) with (3.5)-(3.6) gives

$$
\frac{d}{d t} \int_{0}^{1} \int_{1}^{\infty} \psi_{z} \Phi r^{3} d r d z=\pi^{2} \int_{0}^{1} \int_{1}^{\infty} u^{2} \phi r^{3} d r d z .
$$



yield

Multiplying the first equation in (1.1) with $\nu=0$ by $\Phi$ and integrating on $\Omega(1, \infty)$

$$
\frac{d}{d t} \int_{0}^{1} \int_{1}^{\infty}\left(\log u^{2}\right) \Phi r^{3} d r d z=4 \int_{0}^{1} \int_{1}^{\infty} \psi_{z} \Phi r^{3} d r d z
$$

Since $\int_{0}^{1} \int_{1}^{\infty} \partial_{z} \psi_{0} \Phi r^{3} d r d z>0$, we conclude from (3.9) that

$$
\int_{0}^{1} \int_{1}^{\infty} \psi_{z} \Phi r^{3} d r d z>0 \text { for all } t \geq 0
$$

It follows form ( $(3.10)-(3.11)$ and the condition $\int_{0}^{1} \int_{1}^{\infty}\left(\log u_{0}^{2}\right) \Phi r^{3} d r d z>0$ that

$$
\int_{0}^{1} \int_{1}^{\infty}\left(\log u^{2}\right) \Phi r^{3} d r d z>0 \quad \text { for all } t \geq 0 .
$$

Consequently, we obtain by using (3.2) and (3.12) that

$$
\begin{aligned}
& \left(\int_{0}^{1} \int_{1}^{\infty}\left(\log u^{2}\right) \Phi r^{3} d r d z\right)^{2} \leq\left(\int_{0}^{1} \int_{1}^{\infty}\left(\log ^{+} u^{2}\right) \Phi r^{3} d r d z\right)^{2} \\
& \leq 4\left(\int_{0}^{1} \int_{1}^{\infty}|u| \Phi r^{3} d r d z\right)^{2} \\
& \leq 4 \int_{0}^{1} \int_{1}^{\infty}|u|^{2} \phi r^{3} d r d z \int_{0}^{1} \int_{1}^{\infty}\left(\frac{\Phi}{\phi}\right)^{2} \phi r^{3} d r d z \\
& \leq \frac{8 c_{0} \pi^{2}}{3} \int_{0}^{1} \int_{1}^{\infty}|u|^{2} \phi r^{3} d r d z
\end{aligned}
$$

where $c_{0}$ is defined by

$$
c_{0}=\frac{3}{2 \pi^{2}} \int_{0}^{1} \int_{1}^{\infty}\left(4 \alpha^{2} r^{2}-\left(8 \alpha+\pi^{2}\right)\right)^{2} \phi r^{3} d r d z<\infty .
$$

Using (3.9), (3.10) and (3.13), we get

$$
\begin{aligned}
\frac{d^{2}}{d t^{2}} \int_{0}^{1} \int_{1}^{\infty}\left(\log u^{2}\right) \Phi r^{3} d r d z & =4 \pi^{2} \int_{0}^{1} \int_{1}^{\infty} u^{2} \phi r^{3} d r d z \\
& \geq \frac{3}{2 c_{0}}\left(\int_{0}^{1} \int_{1}^{\infty}\left(\log u^{2}\right) \Phi r^{3} d r d z\right)^{2}
\end{aligned}
$$

Let

$$
Y(t) \equiv \int_{0}^{1} \int_{1}^{\infty}\left(\log u^{2}\right) \Phi r^{3} d r d z
$$

Then (3.15) implies

$$
Y^{\prime \prime}(t) \geq \frac{3}{2 c_{0}} Y(t) .
$$


It follows from (3.10) - (3.11) that $Y^{\prime}(t)>0$. Multiplying (3.17) by $Y^{\prime}(t)$ and integrating in time from 0 to $t$, we get

$$
\begin{aligned}
& \left(Y^{\prime}(t)\right)^{2} \geq\left(Y^{\prime}(0)\right)^{2}-\frac{1}{c_{0}} Y(0)^{3}+\frac{1}{c_{0}} Y(t)^{3} \\
& =\left(4 \int_{0}^{1} \int_{1}^{\infty} \partial_{z} \psi_{0} \Phi r^{3} d r d z\right)^{2}-\frac{1}{c_{0}}\left(\int_{0}^{1} \int_{1}^{\infty}\left(\log u_{0}^{2}\right) \Phi r^{3} d r d z\right)^{3}+\frac{1}{c_{0}} Y(t)^{3} .
\end{aligned}
$$

The condition (2.8) implies

$$
\left(Y^{\prime}(t)\right)^{2} \geq \frac{1}{c_{0}} Y(t)^{3}
$$

Since $Y^{\prime}(t)>0$, it is easy to solve (3.18) to conclude that

$$
\int_{0}^{1} \int_{1}^{\infty}\left(\log u^{2}\right) \Phi r^{3} d r d z \geq \frac{4 c_{0} \int_{0}^{1} \int_{1}^{\infty}\left(\log u_{0}^{2}\right) \Phi r^{3} d r d z}{\left(2 \sqrt{c_{0}}-t \sqrt{\int_{0}^{1} \int_{1}^{\infty}\left(\log u_{0}^{2}\right) \Phi r^{3} d r d z}\right)^{2}}
$$

Note that (3.2) gives

$$
\Phi(r, z)=\left[4 \alpha^{2} r^{2}-\left(8 \alpha+\pi^{2}\right)\right] \phi(r, z)=\left[4 \alpha^{2} r^{2}-\left(8 \alpha+\pi^{2}\right)\right] e^{-\alpha r^{2}} \sin (\pi z) .
$$

Since $r \geq 1$, it is easy to show that $\Phi$ satisfies

$$
0 \leq \Phi(r, z) \leq 4 \alpha^{2} r^{2} e^{-\alpha r^{2}} \leq 4 \alpha^{2} e^{-\alpha}, \quad r \geq 1
$$

Thus, we have

$$
\begin{aligned}
\int_{0}^{1} \int_{1}^{\infty}\left(\log u^{2}\right) \Phi r^{3} d r d z & \leq \int_{0}^{1} \int_{1}^{\infty}\left(\log ^{+} u^{2}\right) \Phi r^{3} d r d z \\
& \leq 4 \alpha^{2} e^{-\alpha} \int_{0}^{1} \int_{1}^{\infty} u^{2} r^{3} d r d z
\end{aligned}
$$

Combining (3.19) with $(3.22)$ gives

$$
4 \alpha^{2} e^{-\alpha} \int_{0}^{1} \int_{1}^{\infty} u^{2} r^{3} d r d z \geq \frac{4 c_{0} \int_{0}^{1} \int_{1}^{\infty}\left(\log u_{0}^{2}\right) \Phi r^{3} d r d z}{\left(2 \sqrt{c_{0}}-t \sqrt{\int_{0}^{1} \int_{1}^{\infty}\left(\log u_{0}^{2}\right) \Phi r^{3} d r d z}\right)^{2}} .
$$

On one hand, we have $\int_{0}^{1} \int_{1}^{\infty} u^{2} r^{3} d r d z<\infty$ for all times by (3.1). On the other hand, the right hand side of (3.23) will blow up as $t \rightarrow \frac{2 \sqrt{c_{0}}}{\sqrt{\int_{0}^{1} \int_{0}^{\infty}\left(\log u_{0}^{2}\right) \Phi r^{3} d r d z}}$. This is clearly a contradiction. This contradiction implies that the assumption (3.1) can not be valid and the solution must blow up in a finite time in the $H^{3}$ norm no later than $T^{*}=\frac{2 \sqrt{c_{0}}}{\sqrt{\int_{0}^{1} \int_{0}^{\infty}\left(\log u_{0}^{2}\right) \Phi r^{3} d r d z}}$. This completes the proof of Theorem 2.2. 


\section{Global Regularity with Large Data}

This section is devoted to proving Theorem 2.5.

Proof of Theorem 2.5. First of all, the local well-posedness of the initial boundary value problem can be established by using an argument similar to that of 24. Now let us assume that $(u, \psi)$ is a local smooth solution satisfying boundary condition (2.17) such that $u \in C\left([0, T) ; H^{s}(\Omega(0,1))\right), \psi \in C\left([0, T) ; H^{s+1}(\Omega(0,1))\right)$ for some $T>0$. We are going to show that $T=\infty$.

Denote by

$$
\widetilde{v}=-\partial_{z} \psi, \quad \widetilde{u}=u^{2}
$$

It is easy to see that

$$
\widetilde{v} \in C\left([0, T) ; H^{s}(\Omega(0,1))\right), \quad \widetilde{u} \in C\left([0, T) ; H^{s}(\Omega(0,1))\right) .
$$

Differentiating the second equation in (1.1) with $\nu=0$ with respect to $z$, we get

$$
\left(\partial_{r}^{2}+\frac{3}{r} \partial_{r}+\partial_{z}^{2}\right) \partial_{t} \widetilde{v}=\partial_{z}^{2} \widetilde{u}
$$

Note that $\left.\partial_{t} \widetilde{v}\right|_{\partial \Omega(0,1)}=0$. We define $g \equiv \Delta_{5}^{-1} f$ as the solution of the Laplacian equation with the homogeneous Dirichlet boundary condition:

$$
\left(\sum_{i=1}^{4} \partial_{x_{i}}^{2}+\partial_{z}^{2}\right) g=f,\left.\quad g\right|_{\partial \Omega(0,1)}=0, \quad \forall f \in L^{2}(\Omega(0,1)) .
$$

Then, we can reformulate the 3D inviscid model as follows:

$$
\left\{\begin{array}{l}
\widetilde{u}_{t}=-4 \widetilde{u} \widetilde{v} \\
\widetilde{v}_{t}=\Delta_{5}^{-1} \partial_{z}^{2} \widetilde{u}
\end{array}\right.
$$

Note that the boundary condition in (2.17) implies that

$$
\left.\widetilde{v}\right|_{\partial \Omega(0,1)}=M \text {. }
$$

If we further denote

$$
v=\widetilde{v}-M,
$$

we obtain an equivalent system for $\widetilde{u}$ and $v$ :

$$
\begin{aligned}
& \widetilde{u}_{t}=-4 M \widetilde{u}-4 \widetilde{u} v, \\
& v_{t}=\Delta_{5}^{-1} \partial_{z}^{2} \widetilde{u}, \\
& \left.v\right|_{\partial \Omega(0,1)}=0 .
\end{aligned}
$$

Recall the following well-known Sobolev inequality [15]. Let $u, v \in H^{s}(\Omega)$ with $s>n / 2(n$ is the dimension of $\Omega)$. We have

$$
\|u v\|_{H^{s}(\Omega)} \leq C_{s}\|u\|_{H^{s}(\Omega)}\|v\|_{H^{s}(\Omega)} .
$$


We will also use the Poincaré inequality [13]:

$$
\|v\|_{H^{s}(\Omega)} \leq C_{s}\|\nabla v\|_{H^{s-1}(\Omega)},
$$

if $\left.v\right|_{\partial \Omega(0,1)}=0$. Here $C_{s}$ is an absolute positive constant depending on $s$ and $\Omega$ only.

Taking the $H^{s}$ norm to the both sides of (4.2) and using (4.5)-(4.6), we obtain

$$
\begin{aligned}
\frac{d}{d t}\|\widetilde{u}\|_{H^{s}} & \leq-4 M\|\widetilde{u}\|_{H^{s}}+4 C_{s}\|\widetilde{u}\|_{H^{s}}\|v\|_{H^{s}} \\
& \leq-4 M\|\widetilde{u}\|_{H^{s}}+4 C_{s}^{2}\|\widetilde{u}\|_{H^{s}}\|\nabla v\|_{H^{s-1}} \\
& \leq\left(-4 M+4 C_{s}^{2}\|\nabla v\|_{H^{s-1}}\right)\|\widetilde{u}\|_{H^{s}}
\end{aligned}
$$

where we have used (4.4).

Next, we apply $\nabla$ to the both sides of (4.3) and take the $H^{s-1}$-norm. We get

$$
\begin{aligned}
\frac{d}{d t}\|\nabla v\|_{H^{s-1}} & \leq\left\|\nabla \Delta_{5}^{-1} \partial_{z}^{2} \widetilde{u}\right\|_{H^{s-1}} \\
& \leq\left\|\Delta_{5}^{-1} \partial_{z}^{2} \widetilde{u}\right\|_{H^{s}} \\
& \leq C_{s}\left\|\partial_{z}^{2} \widetilde{u}\right\|_{H^{s-2}} \leq C_{s}\|\widetilde{u}\|_{H^{s}},
\end{aligned}
$$

where we have used the standard elliptic regularity estimate for $\Delta_{5}^{-1}$.

By the local well-posedness result and the assumption on the initial condition (2.18), we know that there exists a positive $T>0$ such that we have

$$
\|\nabla v(t)\|_{H^{s-1}} \leq \frac{M}{2 C_{s}^{2}}, \quad 0 \leq t \leq T .
$$

Let $T^{*}$ be the largest time such that (4.9) holds. We will prove that $T^{*}=\infty$. Suppose $T^{*}<\infty$. Substituting (4.9) into (4.7), we obtain

$$
\frac{d}{d t}\|\widetilde{u}\|_{H^{s}} \leq-2 M\|\widetilde{u}\|_{H^{s}}, \quad 0 \leq t<T^{*},
$$

which implies

$$
\|\widetilde{u}\|_{H^{s}} \leq\left\|\widetilde{u_{0}}\right\|_{H^{s}} e^{-2 M t}, \quad 0 \leq t<T^{*} .
$$

Now substituting (4.11) into (4.8) yields

$$
\begin{aligned}
\|\nabla v\|_{H^{s-1}} & \leq\left\|\nabla v_{0}\right\|_{H^{s-1}}+C_{s}\left\|\widetilde{u_{0}}\right\|_{H^{s}} \int_{0}^{t} e^{-2 M s} d s \\
& \leq\left\|\nabla v_{0}\right\|_{H^{s-1}}+\frac{C_{s}}{2 M}\left\|\widetilde{u_{0}}\right\|_{H^{s}} \\
& \leq \frac{M}{4 C_{s}^{2}}<\frac{M}{2 C_{s}^{2}}, \quad 0 \leq t<T^{*},
\end{aligned}
$$

where we have used the condition (2.18). Since $\|\nabla v\|_{H^{s-1}} \leq \frac{M}{4 C_{s}^{2}}<\frac{M}{2 C_{s}^{2}}$ for $t<T^{*}$ and $\|\widetilde{u}\|_{H^{s}} \leq\left\|\widetilde{u_{0}}\right\|_{H^{s}} e^{-2 M T^{*}}<\left\|\widetilde{u_{0}}\right\|_{H^{s}}$ for $t<T^{*}$, we can use our a priori estimates (4.7)-(4.8) to show that (4.9) remains valid for $0 \leq t \leq T^{*}+\delta$ for some $\delta>0$. This contradicts with the assumption that $T^{*}$ is the largest time such that (4.9) holds. This contradiction implies that $T^{*}=\infty$ and estimates (4.11)-(4.12) remain valid for all times. This completes the proof of Theorem 2.5. 


\section{Local Well-Posedness in the Exterior Domain}

The local well-posedness theory of the initial-boundary value problem of the 3D model (1.1) with (2.2) and (2.4) is based on the following lemma 5.1. Once the lemma is proved, the proof can be carried out in exactly the same way as the local well-posedness analysis presented in [24]. Hence, we will only present the proof of the lemma 5.1 in a slightly modified domain with $1<r<\infty$ and $0<z<\pi$.

Lemma 5.1. For any given $\omega \in H^{s-2}(\Omega(1, \infty))$ with $s \geq 2$, there exists a unique solution $\psi \in H^{s}(\Omega(1, \infty))$ to the boundary value problems

$$
\left\{\begin{array}{l}
-\left(\partial_{r}^{2}+\frac{3}{r} \partial_{r}+\partial_{z}^{2}\right) \psi=\omega, \quad 1<r, 0<z<\pi \\
\psi_{r}+\left.\beta \psi\right|_{r=1}=0,\left.\quad \psi_{z}\right|_{z=0}=\left.\psi_{z}\right|_{z=\pi}=0
\end{array}\right.
$$

where $\beta \in S_{\text {exterior }}$ is a constant. Furthermore, we have the following estimate:

$$
\|\psi\|_{H^{s}(\Omega(1, \infty))} \leq C\|\omega\|_{H^{s-2}(\Omega(1, \infty))},
$$

where $C$ is an absolute positive constant.

Proof. Let us decompose $\psi=\psi^{(1)}+\psi^{(2)}$, where $\psi^{(1)}$ is the solution of the elliptic equation with the following mixed Dirichlet-Neumann boundary condition:

$$
\left\{\begin{array}{l}
-\left(\partial_{r}^{2}+\frac{3}{r} \partial_{r}+\partial_{z}^{2}\right) \psi^{(1)}=\omega, \quad \text { in } \Omega(1, \infty), \\
\left.\psi_{z}^{(1)}\right|_{z=0}=\left.\psi_{z}^{(1)}\right|_{z=\pi}=0,\left.\quad \psi^{(1)}\right|_{r=1}=0, \quad \psi^{(1)} \rightarrow 0 \text { as } r \rightarrow \infty .
\end{array}\right.
$$

Then $\psi^{(2)}$ satisfies

$$
\left\{\begin{array}{l}
-\left(\partial_{r}^{2}+\frac{3}{r} \partial_{r}+\partial_{z}^{2}\right) \psi^{(2)}=0, \quad \text { in } \Omega(1, \infty) \\
{\left.\left[\psi_{r}^{(2)}+\beta \psi^{(2)}\right]\right|_{r=1}=-\left.\psi_{r}^{(1)}\right|_{r=1}} \\
\left.\psi_{z}^{(2)}\right|_{z=0}=\left.\psi_{z}^{(2)}\right|_{z=\pi}=0 .
\end{array}\right.
$$

The standard elliptic theory gives that $\psi^{(1)} \in H^{s}(\Omega(1, \infty))$ and $\left\|\psi^{(1)}\right\|_{H^{s}(\Omega(1, \infty))} \leq$ $\|\omega\|_{H^{s-2}(\Omega(1, \infty))}$. It remains to show that the Neumann-Robin problem (5.4) is wellposed.

Let us perform the cosine transform to (5.4) with respect to $z$ variable, which gives

$$
\left\{\begin{array}{l}
-\left(\partial_{r}^{2}+\frac{3}{r} \partial_{r}\right) \widehat{\psi^{(2)}}+k^{2} \widehat{\psi^{(2)}}=0 \\
{\left.\left[\psi_{r}^{(2)}+\beta \widehat{\psi^{(2)}}\right]\right|_{r=1}=-\beta \widehat{\psi_{r}^{(1)}}(1, k)}
\end{array}\right.
$$

where the cosine transform $\hat{\imath}$ is defined as:

$$
\widehat{\psi^{(2)}}(r, k)=\frac{2}{\pi} \int_{0}^{\pi} \psi^{(2)}(r, z) \cos (k z) d z .
$$


Let $\widehat{\psi^{(2)}}(r, k)=\frac{1}{r} f(r k, k)$. Then (5.5) can be written in terms of $f$ as

$$
k^{2} \frac{d^{2}}{d r^{2}} f(r k)+\frac{k}{r} \frac{d}{d r} f(r k)-\left(k^{2}+\frac{1}{r^{2}}\right) f(r k)=0,
$$

which is equivalent to the following modified Bessel equation

$$
r^{2} f^{\prime \prime}(r, k)+r f^{\prime}(r, k)-\left(1+r^{2}\right) f(r, k)=0 .
$$

The general solution of the modified Bessel equation with the decaying property as $r \rightarrow \infty$ is given by modified Bessel function:

$$
f(r, k)=C(k) K(r)
$$

where $K(r)$ is the modified Bessel function and can be represented in an integration form as follows

$$
K(r)=\int_{0}^{\infty} e^{-r \cosh (\theta)} \cosh (\theta) d \theta, \quad K(r) \rightarrow 0 \text { as } r \rightarrow \infty
$$

In fact, we have $0<K(r) \leq c_{2} r^{-1 / 2} e^{-r}$ as $r \rightarrow \infty$ for some positive constant $c_{2}$. Thus, the general solution of $\widehat{\psi^{(2)}}(k, r)$ is given by

$$
\widehat{\psi^{(2)}}(r, k)=\frac{C(k)}{r} K(r k) .
$$

The boundary condition of $\psi^{(2)}$ in $(5.5)$ can be used to determine $C(k)$ as follows:

$$
C(k)=\frac{-\beta \widehat{\psi^{(1)}}(k, 1)}{(\beta-1) K(k)+k K^{\prime}(k)} .
$$

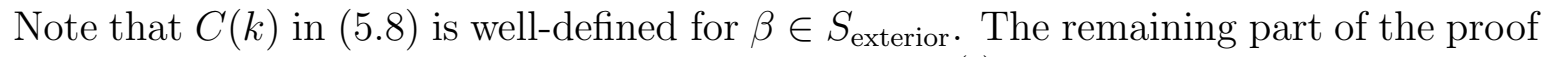
is exactly the same as in [24]. We can show that $\psi^{(2)}$ will have the same regularity property as $\psi^{(1)}$. This proves our lemma. We will omit the details here.

\section{Acknowledgement}

This work was supported in part by NSF by Grant DMS-0908546. Zhen Lei would like to thank the Applied and Computational Mathematics of Caltech and Prof. Thomas Hou for hosting his visit and for their hospitality during his visit. Zhen Lei was in part supported by NSFC (grant No.11171072), the Foundation for Innovative Research Groups of NSFC (grant No.11121101), FANEDD, Innovation Program of Shanghai Municipal Education Commission (grant No.12ZZ012) and SGST 09DZ2272900. The research of Dr. S. Wang was supported by China 973 Program(Grant no. 2011CB808002), the Grants NSFC 11071009 and phr-ihlb 2009061p3. 


\section{References}

[1] Bardos, C.; Titi, E. S.; Euler equations for incompressible ideal fluids, Uspekhi Mat. Nauk, 62(3) 375 (2007), 5-46

[2] Beale, J. T.; Kato, T.; Majda, A. Remarks on the breakdown of smooth solutions for the 3-D Euler equations. Comm. Math. Phys. 94 (1984), no. 1, 61-66.

[3] Boratav, O. N.; Pelz, R. B.; Direct numerical simulation of transition to turbulence from a high-symmetry initial condition. Phys. Fluids 6 (1994), 2757-2784.

[4] Caffarelli, L.; Kohn, R.; Nirenberg, L. Partial regularity of suitable weak solutions of the Navier-Stokes equations. Comm. Pure Appl. Math. 35 (1982), 771-831.

[5] Caflisch, R.; Siegel, M; A semi-analytic approach to Euler singularities, Methods and Appl. of Analysis. 11 (2004), 423-430.

[6] Chae, D.; On the finite-time singularities of the 3D incompressible Euler equations, Comm. Pure Appl. Math. 60 (2007), 597-617.

[7] Constantin, P.; Note on loss of regularity for solutions of the 3D incompressible Euler and related equations, Commun. Math. Phys. 104 (1986), 311-326.

[8] Constantin, P.; On the Euler equations of incompressible fluids, Bull. Amer. Math. Soc., 44 (2007), 603-621.

[9] Constantin, P.; Fefferman, C.; Majda, A.; Geometric constraints on potentially singular solutions for the 3-D Euler equation, Comm. in PDEs. 21 (1996), 559571.

[10] Cordoba, D.; Fefferman, C.; On the collapse of tubes carried by 3D incompressible flows. Comm. Math. Phys. 222 (2001), 293-298.

[11] Deng, J.; Hou, T. Y.; Yu, X.; Geometric properties and non-blowup of 3-D incompressible Euler flow, Comm. in PDEs. 30 (2005), 225-243.

[12] Deng, J.; Hou, T. Y.; Yu, X.; Improved geometric conditions for non-blowup of 3D incompressible Euler equation, Comm. in PDEs. 31 (2006), 293-306.

[13] L. C. Evans, Partial Differential Equations, American Mathematical Society Publ., 1998.

[14] Fefferman, C. Available online at http://www.claymath.org/millennium/NavierStokes equations.

[15] Foland, G. B. Introduction to Partial differential Equations, Princeton University Press, Princeton, N.J., 1995 
[16] Grauer, R.; Sideris, T.; Numerical computation of three dimensional incompressible ideal fluids with swirl. Phys. Rev. Lett. 67 (1991), 3511-3514.

[17] Hou, T. Y.; Blow-up or No Blow-up? A unified computational and analytic approach to study 3-D incompressible Euler and Navier-Stokes equations, Acta Numerica, 18 (2009), 277-346.

[18] Hou, T. Y.; Li, R.; Computing nearly singular solutions using pseudo-spectral methods. J. Comput. Phys. 226 (2007), 379-397.

[19] Hou, T. Y.; Li, R.; Dynamic depletion of vortex stretching and non-blowup of the 3-D incompressible Euler equations. J. Nonlinear Science. 16 (2006), no. 6, 639-664.

[20] Hou, T. Y.; Li, R. Computing nearly singular solutions using pseudo-spectral methods. J. Comput. Phys. 226 (2007), 379-397.

[21] Hou, T. Y.; Li, C.-M. Dynamic stability of the 3D axi-symmetric Navier-Stokes equations with swirl. Comm. Pure Appl. Math. 61 (2008), no. 5, 661-697.

[22] Hou, T. Y.; Lei, Z. On the Stabilizing Effect of Convection in Three-Dimensional Incompressible Flows. Comm. Pure Appl. Math. 62 (2009), no. 4, 502-564.

[23] Hou, T. Y.; Lei, Z. On partial regularity of a 3D model of Navier-Stokes equations. Comm. Math. Phys. 287 (2009), 281-298.

[24] Hou, T. Y.; Shi, Z.; Wang, S. On singularity formation of a 3D model for incompressible Navier-Stokes equations. Adv. Math. (2012), accepted for publication.

[25] Lei, Z; Lin, F.-H. Global Mild Solutions of the Navier-Stokes Equations, Communications on Pure and Applied Mathematics, 64 (2011), no. 9, 1297-1304.

[26] Ladyzhenskaya, O. A. Mathematical Questions of the Dynamics of a Viscous Incompressible Fluid. Nauka, Moscow, 1970.

[27] Kerr, R. M.; Evidence for a singularity of the three dimensional, incompressible Euler equations. Phys. Fluids 5 (1993), no. 7, 1725-1746.

[28] Lin, F.-H. A new proof of the Caffarelli-Kohn-Nirenberg theorem. Comm. Pure Appl. Math. 51 (1998), no. 3, 241-257.

[29] Majda, A. J.; Bertozzi, A. L. Vorticity and incompressible flow. Cambridge Texts in Applied Mathematics, 27. Cambridge University Press, Cambridge, 2002.

[30] Prodi, G. Un teorema di unicità per le equazioni di Navier-Stokes.Ann. Mat. Pura Appl. 48 (1959), 173-182.

[31] Serrin, J. The initial value problem for the Navier-Stokes equations. Nonlinear Problems, Univ. of Wisconsin Press, Madison, 1963, 69-98. 